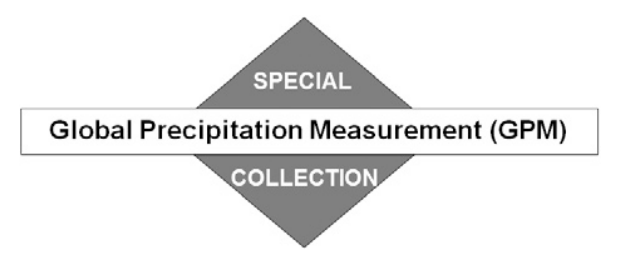

\title{
TRMM Microwave Imager (TMI) Alignment and Along-Scan Bias Corrections
}

\author{
RACHAEL KROODSMA \\ Earth System Science Interdisciplinary Center, University of Maryland, College Park, College Park, \\ and NASA Goddard Space Flight Center, Greenbelt, Maryland \\ STEPHEN BILANOW \\ SGT Inc., and NASA Goddard Space Flight Center, Greenbelt, Maryland \\ DARREN MCKAGUE \\ University of Michigan, Ann Arbor, Michigan
}

(Manuscript received 20 December 2017, in final form 20 April 2018)

\begin{abstract}
The Tropical Rainfall Measuring Mission (TRMM) Microwave Imager (TMI) dataset released by the Precipitation Processing System (PPS) has been updated to a final version following the decommissioning of the TRMM satellite in April 2015. The updates are based on increased knowledge of radiometer calibration and sensor performance issues. In particular, the Global Precipitation Measurement (GPM) Microwave Imager (GMI) is used as a model for many of the TMI updates. This paper discusses two aspects of the TMI data product that have been reanalyzed and updated: alignment and along-scan bias corrections. The TMI's pointing accuracy is significantly improved over prior PPS versions, which used at-launch alignment values. A TMI instrument mounting offset is discovered as well as new alignment offsets for the two TMI feedhorns. The original TMI along-scan antenna temperature bias correction is found to be generally accurate over ocean, but a scene temperature-dependent correction is needed to account for edge-of-scan obstruction. These updates are incorporated into the final TMI data version, improving the quality of the data product and ensuring accurate geophysical parameters can be derived from TMI.
\end{abstract}

\section{Introduction}

The Tropical Rainfall Measuring Mission (TRMM) was launched in November 1997 carrying on board the TRMM Microwave Imager (TMI) and Precipitation Radar (PR) as the primary instruments to measure rainfall (Kummerow et al. 1998). After a successful 17-plus years of operation, the spacecraft was decommissioned in April 2015, as the orbit decayed and the spacecraft altitude dropped below $350 \mathrm{~km}$ - a result of the fuel on board running out in July 2014. As part of end-of-mission activities, the TRMM data products have been reanalyzed and updated to a final version 8 (V8), replacing the previous version 7 (V7) data products that were released in 2011 (Stocker et al. 2018). This paper describes two major updates to the TMI V8 data product.

\footnotetext{
Corresponding author: Rachael Kroodsma, rachael.a.kroodsma@ nasa.gov
}

Shortly after TRMM was launched, an extensive postlaunch analysis of the TMI data was performed by Wentz et al. (2001) to correct TMI calibration issues. While microwave radiometers are tested prior to launch, there are many factors that impact the instrument on orbit in the space environment that cannot be accounted for prelaunch, such as solar intrusions (Kunkee et al. 2008), field-of-view obstructions (McKague et al. 2011), thermal variations (Gopalan et al. 2009), and alignment errors (Poe et al. 2008). These issues are identified and corrected postlaunch using the on-orbit data. Since the launch of TRMM, several other radiometers have been launched, leading to a greater understanding of potential radiometer calibration issues and how to correct for them. As a result, radiometer designs have improved and the algorithms to analyze on-orbit data have advanced, as evidenced most recently by the Global Precipitation Measurement (GPM) Microwave Imager (GMI), which is acknowledged as a very stable and 
accurate microwave radiometer as a result of design enhancements and on-orbit data analysis (Draper et al. 2015; Wentz and Draper 2016). The corrections included in TMI V8 are derived based on this increased knowledge of other radiometer calibration issues, and in particular the GMI on-orbit analysis is used as a model for many of the TMI V8 updates.

There are several modifications that are incorporated into TMI V8 to improve the calibration and quality of the data product. This paper discusses two of these modifications: the alignment of the instrument and feedhorns, and the along-scan temperature bias correction. Other corrections to TMI V8 include a hot load correction (Alquaied et al. 2018a), updated emissive antenna correction (Alquaied et al. 2018b), radio frequency interference flag (Draper 2018), improved calibration (Stocker et al. 2018), and intercalibration to GMI (Berg 2017). These corrections are included in the dataset released by the Precipitation Processing System (PPS). Wentz (2015) also provides a thorough analysis of the TMI mission data and derives similar corrections to the TMI data as are incorporated in the PPS TMI V8 dataset. However, Wentz describes corrections to TMI based on the Remote Sensing Systems (RSS) algorithms that are included in the data version released by RSS, whereas the corrections described here are based on PPS algorithms. In this paper we also add an instrument alignment offset to update geolocation and analysis over warm targets to update an antenna temperature dependence of the along-scan bias correction beyond those performed by RSS.

In addition to updating the TMI data product, all spaceborne microwave radiometers with similar channels dating back to December 1997 will be incorporated into the GPM mission's constellation of radiometers. This constellation originally included only those radiometers in operation after the launch of GPM in February 2014 (Hou et al. 2014). Since TMI observations overlap with GMI, TMI can be used to intercalibrate radiometers that existed prior to the launch of GPM, resulting in a consistent dataset of historical observations. The GPM Intercalibration Working Group (XCAL) is responsible for calculating the intercalibration constants used for the dataset (Berg et al. 2016). Since TMI makes up a significant portion of this intercalibration dataset and is used as a transfer for radiometers prior to GMI, the TMI V8 improvements are critical to ensuring an accurate long-term data record that can be reliably used for climate studies.

\section{Instrument description}

TMI was a nine-channel conical scanning microwave radiometer with an offset parabolic reflector viewing about $49^{\circ}$ from nadir and an approximate Earth-view azimuthal rotation scan of $130^{\circ}$ (Kummerow et al. 1998). The design was modeled after the Special Sensor Microwave Imager (SSM/I; Hollinger et al. 1990) and included similar channels to SSM/I centered at 19.35 , $21.3,37.0$, and $85.5 \mathrm{GHz}$ (referred to as $19,21,37$, and $85 \mathrm{GHz}$, respectively). In addition, TMI included a lowerfrequency channel centered at $10.65 \mathrm{GHz}$ to measure heavier rainfall (referred to as $10 \mathrm{GHz}$ ). The $19-85-\mathrm{GHz}$ channels shared a feedhorn, while the $10-\mathrm{GHz}$ channels were contained in a separate feedhorn. All channels measured both vertical and horizontal polarization (v-pol and h-pol, respectively), except $21.3 \mathrm{GHz}$, which was only v-pol. The TMI instrument field of view swept a conical path about the instrument spin axis and sampled 104 pixels across the scan for the $10-37-\mathrm{GHz}$ channels and 208 pixels for the higher-resolution $85-\mathrm{GHz}$ channels. A nominal azimuth start angle of $-64.4024^{\circ}$ for the Earth-view scan was estimated prior to launch. The TMI spin rate of $31.6 \mathrm{rpm}$ was observed to be extremely uniform, and the instrument clock controlled the sampling at even intervals of $3.3 \mathrm{~ms}$, giving a rotation angle of $0.6256^{\circ}$ between the high-frequency samples.

\section{Description of corrections}

\section{a. Alignment}

The alignment parameters that we estimate in this paper encompass offsets in the instrument axes relative to the spacecraft axes and offsets in the scan cone half angle that result from feedhorn offsets. There are four alignment parameters that we adjust: 1) pitch: alignment bias about the instrument pitch axis; 2) roll: alignment bias about the instrument roll axis; 3) azimuth: adjustment of the start of sampling azimuth angle, or instrument yaw; and 4) cone: adjustment of scan cone half angle, or angular radius of scan cone. Items 3 and 4 have the possibility of different values for different channels because of feedhorn offsets. Previous TMI versions used values estimated prior to launch. We also include a time delay between the sampling of the multichannel feedhorn and the $10-\mathrm{GHz}$ feedhorn that was not included in previous data versions. This delay is estimated to be $0.2238 \mathrm{~s}$ based on the angle of rotation between the two feedhorn positions.

\section{b. Along-scan temperature bias}

The first postlaunch analysis of TMI data noticed significant along-scan biases for many of the channels (Wentz et al. 2001). A simple offset versus scan position correction by channel was implemented to remove the biases in the data product. Over-ocean antenna temperatures (TAs) were averaged using various filters to derive a 
scan bias as a difference from the mean. This correction assumes that the scan biases are constant at all scene temperatures; however, recent analysis shows that this assumption is not correct. Similar along-scan biases were noticed in the GMI data shortly after it launched (Draper et al. 2015). There are two components to the GMI alongscan correction: 1) a magnetic interference correction and 2) a residual bias from antenna intrusions that includes a temperature-dependent correction for removing edge-ofscan obstructions. Item 1 was determined using ground measurements, and item 2 was calculated using on-orbit data. Some features in the TMI scan angle dependence may have an explanation similar to that suggested for GMI, but we do not have ground measurements from TMI to separate the two corrections. Therefore, a temperaturedependent along-scan correction is derived for TMI using on-orbit data that encompasses both potential magnetic interference and antenna intrusions.

\section{Procedure}

A complication with updating the TMI dataset is that some corrections are dependent on each other. Corrections were derived in sequence; that is, we first updated two aspects of alignment (cone and azimuth) that impact geolocation and earth incidence angle (EIA). These values are needed to process our radiative transfer model for the along-scan correction. However, after analyzing the along-scan correction, we determined that we needed to further update other aspects of the alignment (pitch and roll), which in turn meant recalculating the alongscan correction. Concurrently, several other groups within the XCAL team and PPS were evaluating various other updates to TMI V8. PPS reprocessed the TMI dataset several times during this revision period to ensure that all the corrections are accurate and complement each other. This section will discuss the procedure for deriving the alignment and along-scan bias corrections, and the following section will present the results of the analysis.

\section{a. Alignment corrections for cone and azimuth angles using geolocation}

Alignment errors show up in their effects on image pixel geolocation. The alignment of TMI is examined by creating maps of gridded TAs for the spacecraft's two yaw orientations. To keep the sun on one side of the spacecraft for thermal control, the TRMM spacecraft yawed $180^{\circ}$ every two to four weeks, thereby switching TMI from a forward- to a backward-looking orientation. The effect of an alignment error on pixel coordinates will generally be different for the forward look of the instrument than for the backward look. There is a large contrast between land and ocean TAs at microwave imager frequencies, so small offsets in geolocation cause coastlines to appear highlighted when taking the difference between the forward- and backward-looking maps of observed TAs. This method has been used with great success for various sun-synchronous orbiters by taking the difference between ascending and descending passes (e.g., Berg et al. 2013; Moradi et al. 2013). With the $98^{\circ}$ inclination of sun-synchronous orbits, the flight directions of the ascending and descending orbits when crossing the equator are nearly opposite. This means various alignment biases have nearly opposite effects on the pixel geolocation. However, for TRMM's non-sunsynchronous $35^{\circ}$ inclination orbit, the difference in flight direction when crossing the equator is approximately $70^{\circ}$. Therefore, TRMM's two yaw orientations are used for alignment analysis, since they have nearly opposite effects from the Yaw 0 versus the Yaw 180 flight (fore vs aft viewing angle) cases.

Figure 1 shows $0.1^{\circ}$ gridded Yaw 0 - Yaw 180 TAs for observations from 2004 using V7 geolocation over South America. The TMI geolocation algorithm is a line-ofsight intersection with a World Geodetic System 1984 (WGS-84) Earth ellipsoid model. Topography is not considered because of the large footprint sizes. This does not impact our geolocation analysis, as we are concerned with only coastlines. The South America region is chosen for analysis because of its proximity around the equator and the variety of coastline directions. Proximity to the equator reduces error resulting from incomplete sampling across the scan at the higher latitudes of the TRMM orbit, and the variety of coastlines ensures that this method is valid for all directions. The coastlines in Fig. 1 are apparent in several channels, most notably in the $10 \mathrm{~h}$ channel. This indicates that the $\mathrm{V} 7$ alignment is incorrect and needs to be updated. The TRMM spacecraft attitude is tracked to about $0.01^{\circ}$ as described in Stocker et al. (2018); therefore, this alignment error is attributed to the TMI instrument.

We chose to adjust the cone and azimuth angles as the first steps. In a rough sense the cone bias adjusts the coastline forward and back relative to the ground track, while the azimuth bias adjusts it left and right, so these two effects are fairly independent. To quantitatively assess the optimal cone and azimuth angles, a coast mask is applied to the Yaw 0 - Yaw 180 maps and the root-mean-square (RMS) of the TA difference is calculated, as shown in Fig. 2 for the $10-\mathrm{GHz}$ channels. The optimal alignment parameters will make the coastlines disappear as the RMS differences are minimized.

There are four primary steps to derive the alignment parameters: 1) vary the cone or azimuth angles and calculate the latitude and longitude for each TMI pixel, 2) grid the TAs by yaw orientation and calculate the 

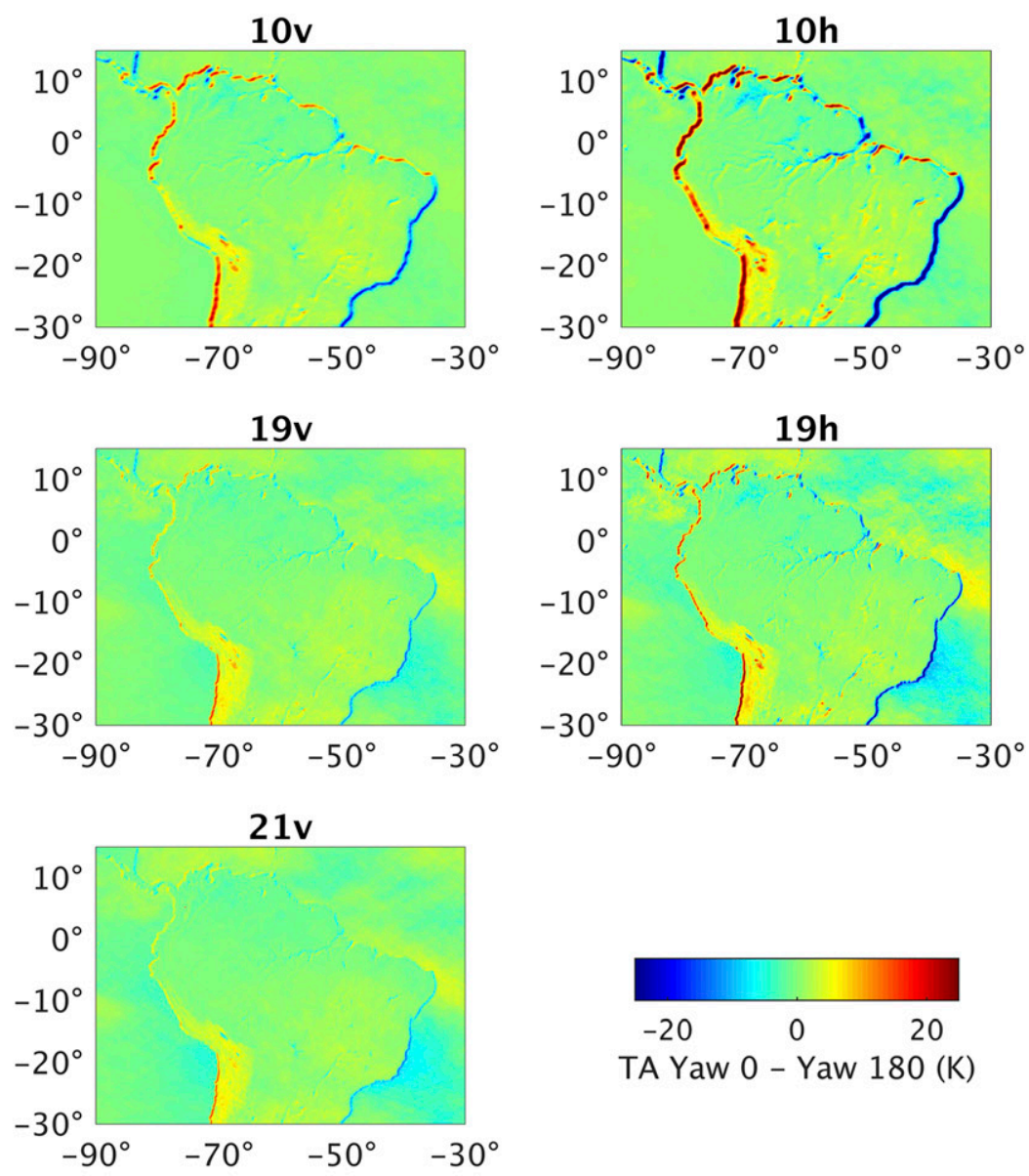

$37 v$
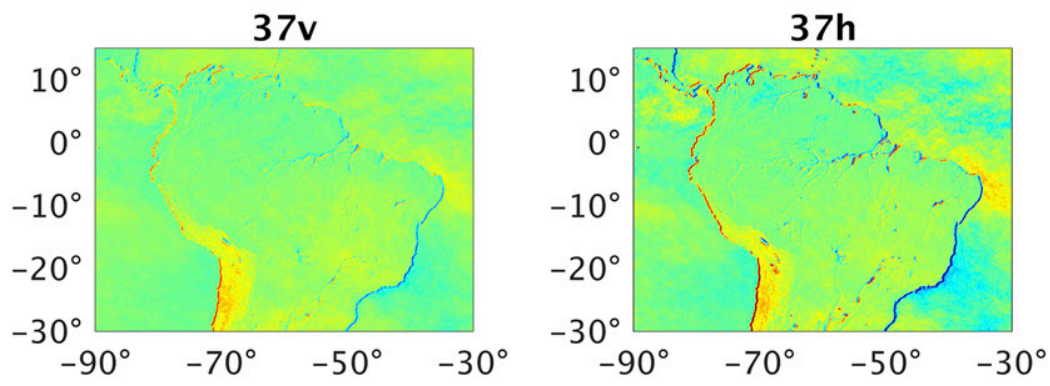

$85 v$
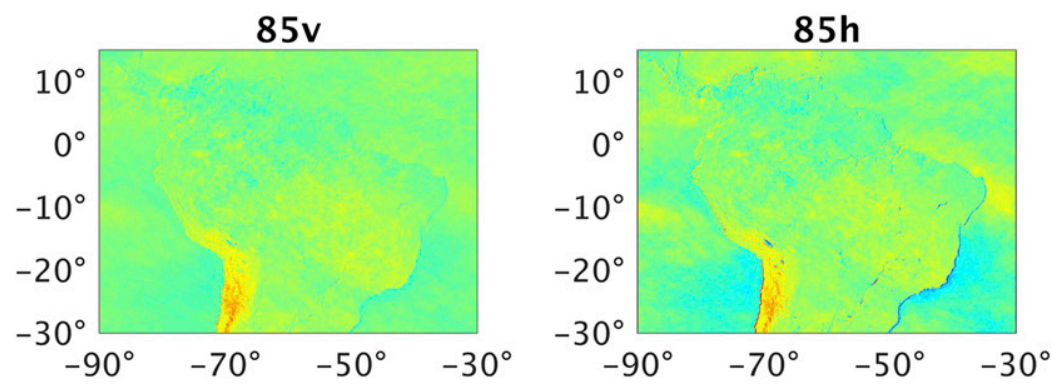

FIG. 1. Yaw 0 - Yaw 180 gridded TMI TAs (K) over South America for V7 geolocation. Incorrect alignment results in the appearance of coastlines, which can be seen in all channels but is most pronounced in the $10 \mathrm{~h}$ channel. 

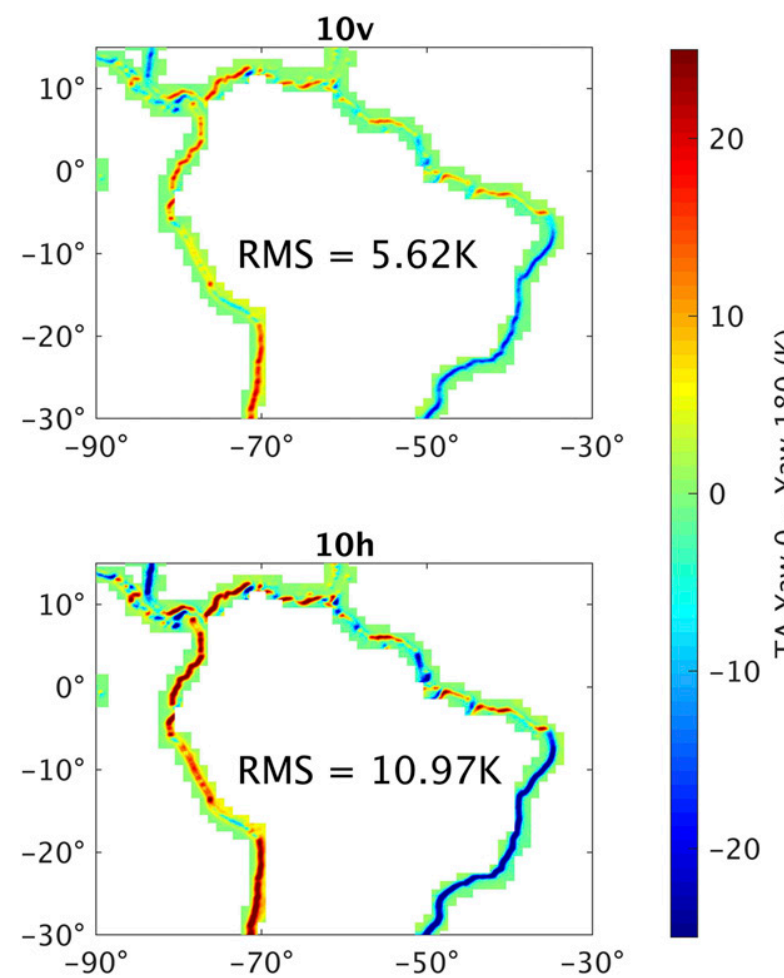

FIG. 2. Coastline mask of Yaw 0 - Yaw 180 gridded TMI TAs (K) used for alignment analysis shown for the 10-GHz channels. The RMS of TA differences contained in the mask is used as a metric for determining optimal alignment values.

Yaw 0 - Yaw 180 TA difference for each cone and azimuth value, 3 ) use the coastline mask to calculate the RMS for each angle, and 4) fit a third-order polynomial to the RMS as a function of cone or azimuth angle to find the angle associated with the minimum RMS. For step 1 , the cone angle is varied from $49.0^{\circ}$ to $49.6^{\circ}$ while holding the azimuth angle constant at $-64.4^{\circ}$, and the azimuth angle is varied from $-64.5^{\circ}$ to $-63.7^{\circ}$ while holding the cone angle constant at $49.0^{\circ}$. This analysis assumes that the cone and azimuth angles are independent of each other, which was found to be a reasonable assumption. For step 2 , a $0.1^{\circ}$ grid size is used. This grid size was determined to be sufficient, since smaller grid sizes did not significantly impact our results and required more data to decrease noise and resulted in increased processing time. Three months of data from January to March 2004 are chosen as the test dataset. We found that this gives adequate sampling while keeping a reasonable processing time. Figure 3 shows the results of step 4 for the $19 \mathrm{v}$ channel, where the RMS for each angle (cone or azimuth) is plotted and a third-order polynomial is fit to the data. The angle where the RMS is at a minimum is chosen as the optimal cone or azimuth angle for that channel.
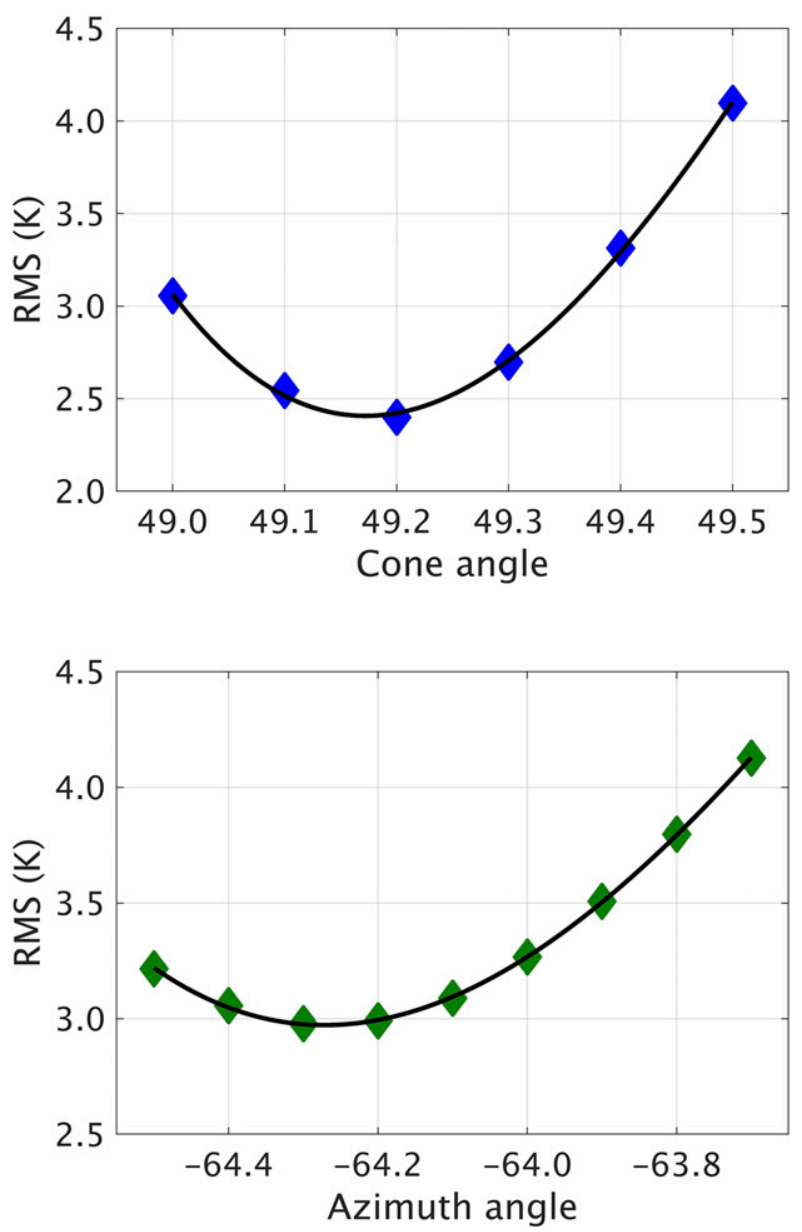

FIG. 3. RMS of the TA difference coastline mask for $19 \mathrm{v}$ as a function of (top) cone and (bottom) azimuth angles. A thirdorder polynomial is fitted to the RMS and the angle where this curve is at a minimum is taken as the optimal cone or azimuth angle for the channel.

\section{b. Along-scan antenna temperature bias correction}

At a given scan position, the observed TA can be decomposed into the desired on-Earth interference-free main-beam brightness temperature (TB) $T_{b, \mathrm{mb}}$, and the contribution from interference or obstruction given the effective brightness of the source of interference $T_{b, i}$ and the effective beam fraction of the interference $f_{i}$ as (McKague et al. 2011)

$$
\mathrm{TA}=T_{b, \mathrm{mb}} \times\left(1-f_{i}\right)+T_{b, i} \times f_{i} .
$$

Estimating $T_{b, i}$ and $f_{i}$ at each scan position requires known measurements $T_{b, \mathrm{mb}}$. This is done by using overocean observations from the vicarious cold calibration technique (Kroodsma et al. 2017) and overland observations from the vicarious warm calibration technique (Yang et al. 2016). These biases can be linearly 
interpolated to compute the bias at an arbitrary scene temperature (McKague et al. 2011; Draper 2016). Since beam patterns as well as sources of interference vary from channel to channel, this is done independently for each channel. Figure 4 shows the TA along-scan biases calculated using the vicarious cold (blue line) and warm (red line) techniques. Both the cold and warm alongscan biases show similar patterns for the large- and small-scale fluctuations, such as the large bump in $21 \mathrm{v}$ and small ripples in $85 \mathrm{~h}$. These fluctuations are most likely due to internal magnetic interference, as they are similar to those seen in GMI. The GMI 10h channel also displayed large bumps in the scan biases like TMI $21 \mathrm{v}$ as a result of that receiver being the closest to magnets on the instrument (Draper et al. 2015). Unfortunately, we do not have enough information about the TMI instrument to reach the same conclusion for why TMI $21 \mathrm{v}$ has the largest scan biases, but we hypothesize a similar reason. As with GMI, the TMI scan fluctuations caused by possible magnetic interference are constant with respect to scene temperature. However, there are two distinct differences in the cold and warm TMI biases. One difference is the edge-of-scan pattern attributed to an obstruction. The second difference is an overall curvature or slope pattern that is different for cold versus warm along-scan biases, most notably in the v-pol channels.

\section{c. Alignment effects on along-scan antenna temperature biases}

Figure 4 shows that one main difference between the cold and warm along-scan biases is an overall curvature and/or slope pattern. We ideally want to understand where this difference comes from and to correct the underlying problem if possible. One phenomenon that can cause some curvature and/or slope changes across the scan is a pitch and/or roll offset of the spacecraft (Kroodsma et al. 2012). Pitch and roll offsets directly affect the EIA of the radiometer, and the over-ocean cold biases are sensitive to EIA variations across the scan, whereas the warm biases are not. Roll changes give a roughly linear slope in EIA with scan angle, while pitch changes give a quadratic-like curvature versus scan angle. By contrast, a cone bias gives a constant EIA offset, while an azimuth bias does not affect the EIA.

Assuming that the large- and small-scale fluctuations in the along-scan biases are constant as a function of scene temperature, taking the difference between the cold and warm scan biases for the middle portion of the scan (to remove edge-of-scan effects) allows a pitch and roll offset to be calculated from the resulting pattern across the scan. Figure 5 shows the warm-minus-cold TA bias difference for the whole scan (black line) as well as a middle portion of the scan (green line). The exact sensitivity of TAs to EIA over cold oceans varies with channel, but roughly the TMI v-pol channels change by $2 \mathrm{~K}$ and $\mathrm{h}$-pol channels by $-1 \mathrm{~K}$ for every $1^{\circ}$ EIA. This behavior is reflected in Fig. 5, where the v-pol channels (left column) all show a similar variation across the scan, whereas the h-pol channels (right column) all exhibit similar but slightly opposite behavior from v-pol. Therefore, we conclude that this difference between cold and warm along-scan biases is due to EIA offsets from a pitch and/or roll offset. A rough evaluation of the mean slopes and curvatures for the middle of the scan in Fig. 5 indicates that a pitch and roll bias on the order of a tenth of degree is needed. To obtain a better estimation of the exact offsets, the method described in the next section was developed.

\section{d. Alignment corrections for pitch and roll offsets using geolocation}

Another method to examine a potential pitch-roll offset is to use the four-step geolocation analysis for calculating the cone angle as outlined in section 4a but to apply the analysis to different sections of the scan instead of using all scan positions together. A cone angle offset causes a constant geolocation shift across the scan, so the derived cone angle is the same at all scan positions. However, a pitch-roll offset results in a different pattern across the scan, as seen in Fig. 6, which models the geolocation of a TMI scan for nominal pointing (no pitch-roll offset, $49^{\circ}$ cone angle), a cone angle offset of $+5^{\circ}$, a pitch offset of $+5^{\circ}$, and a roll offset of $+5^{\circ}$ (these values are exaggerated to see effect). This behavior can be used to distinguish between a pitch-roll offset and the cone angle by applying the geolocation analysis at different scan positions. With the axis of the scan nominally at nadir, the shifts in geolocation from pitch or roll biases give a different cone angle bias at different scan positions. Yaw alignment errors are not considered here, since they have exactly the same effect as the changes in the azimuth angle. Therefore, any yaw errors are incorporated into the updated azimuth start angle.

The geolocation analysis is applied to three subsets of scan positions at the left (10-30 scan positions, low resolution; 20-60 scan positions, high resolution), middle (40-60, 80-120), and right $(70-90,140-180)$ parts of the TMI scan. Using a range of scan positions for each subset allows sufficient sampling, and the far left and right edges of the scan are excluded to remove potential edge-ofscan obstructions. Results are discussed in the next section.

\section{Results}

As noted in the previous section, our process for updating alignment and along-scan bias corrections is 

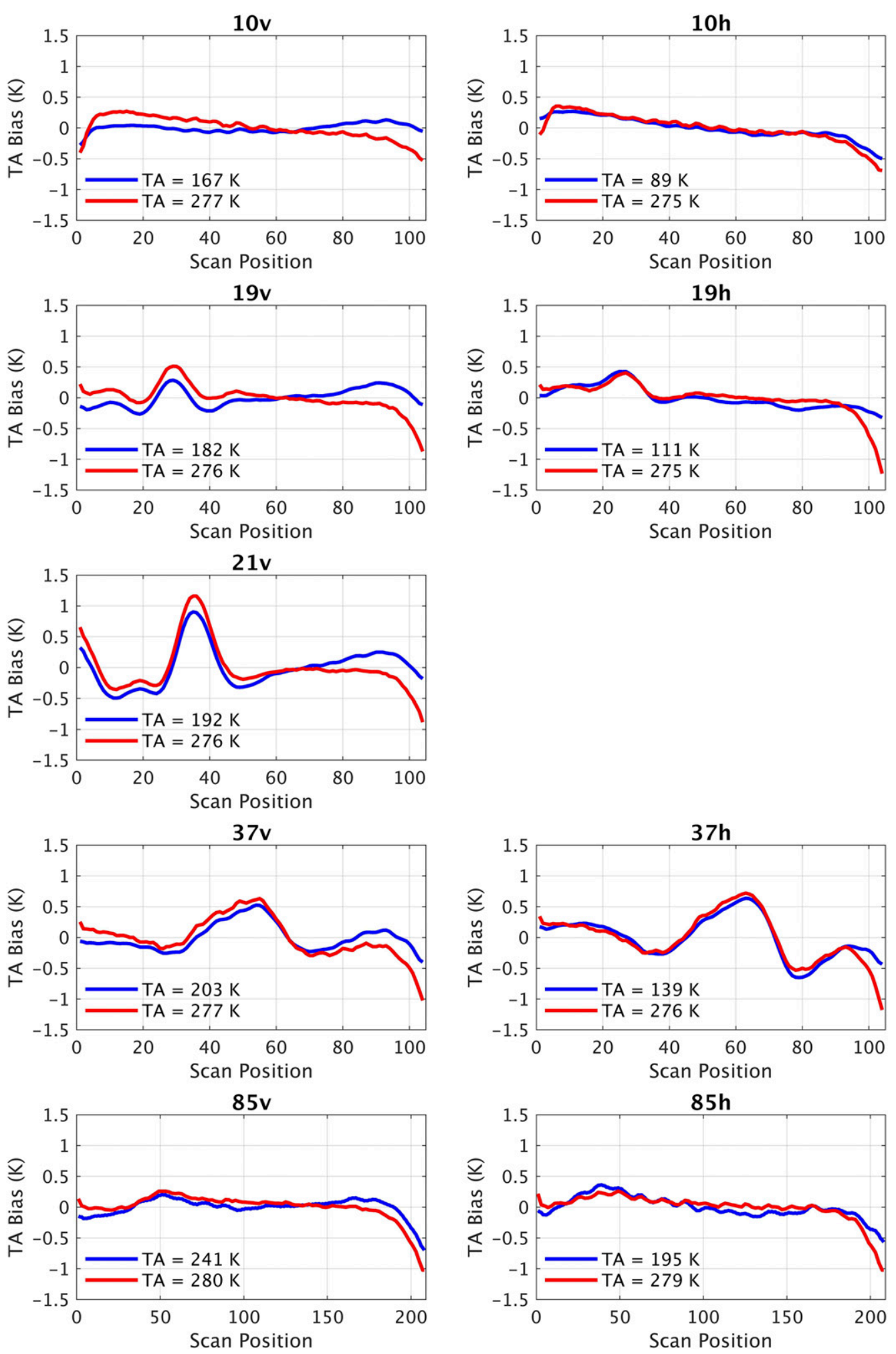

FIG. 4. TMI TA cold (blue) and warm (red) along-scan biases calculated using (top left to bottom right) vicarious calibration techniques. The large- and small-scale fluctuations are similar at both TAs, but the edge-ofscan and overall patterns have some significant differences. 


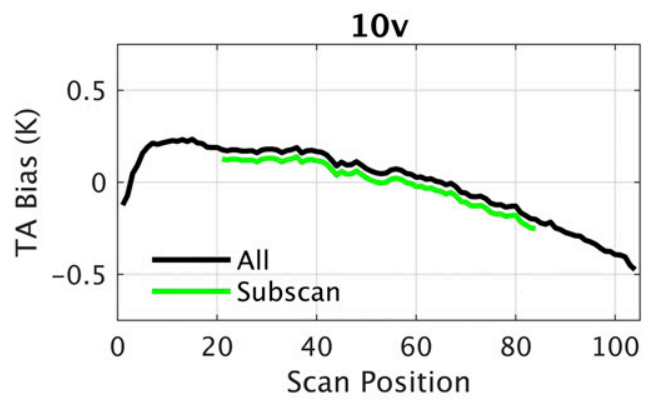

19v

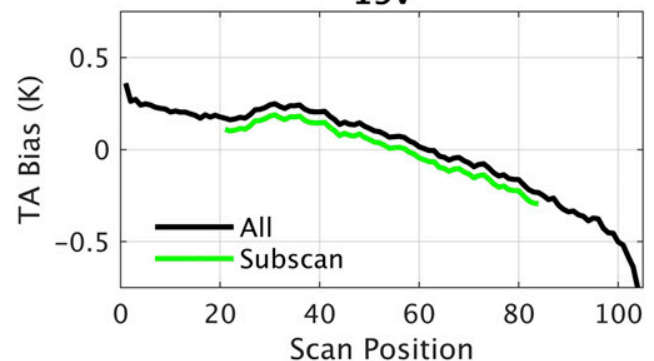

21v

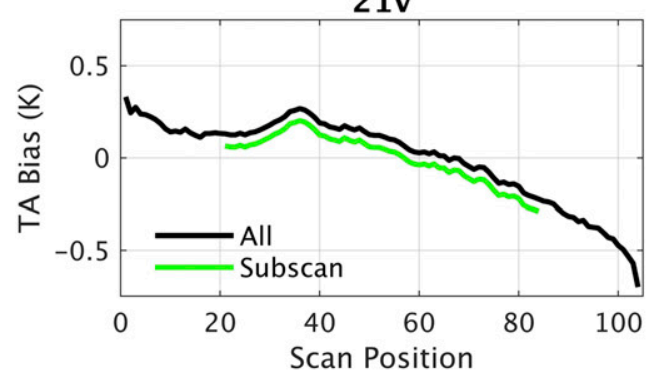

$37 v$

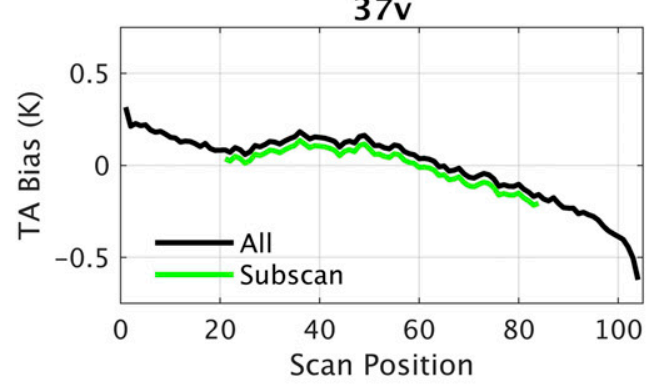

$85 v$

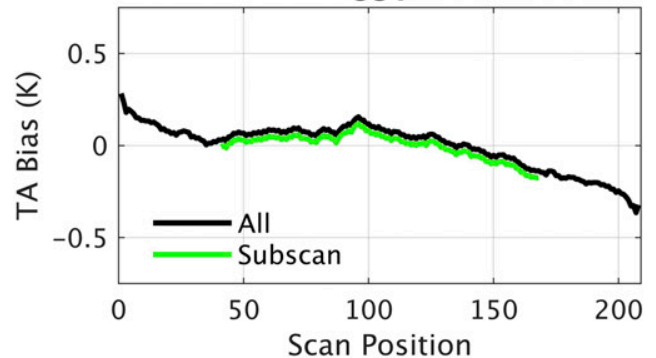

$10 \mathrm{~h}$

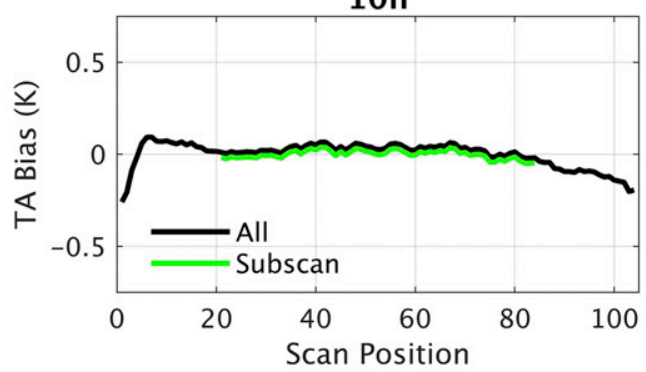

19h

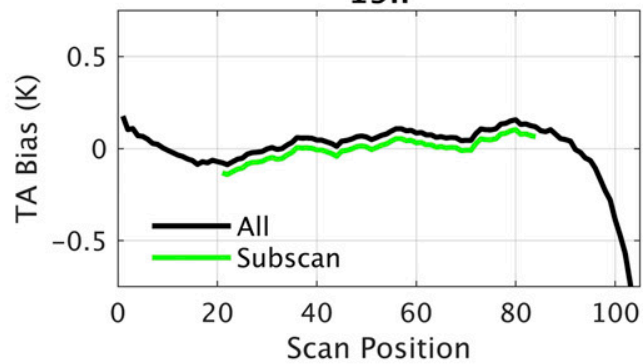

$37 \mathrm{~h}$

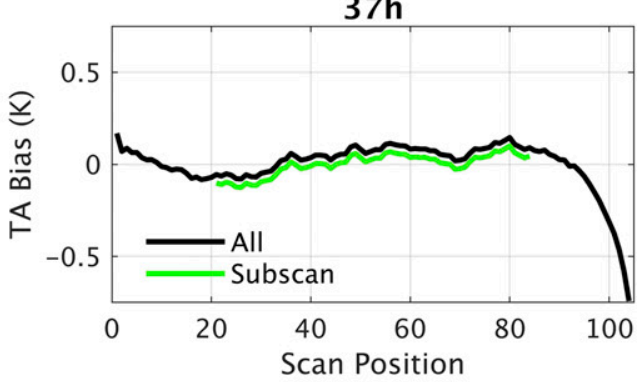

$85 \mathrm{~h}$

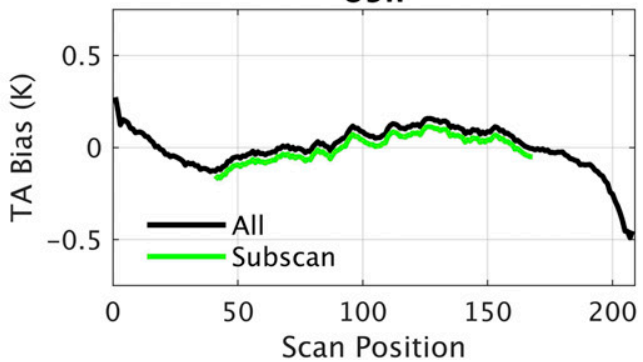

FIG. 5. The TA warm - cold along-scan bias for the whole scan (black line) and the middle of the scan (green line), shown as a difference from the mean for (top left) 10v to (bottom right) 85h. The difference between the (left) v-pol channels and (right) h-pol channels indicates a pitch and/or roll offset. 

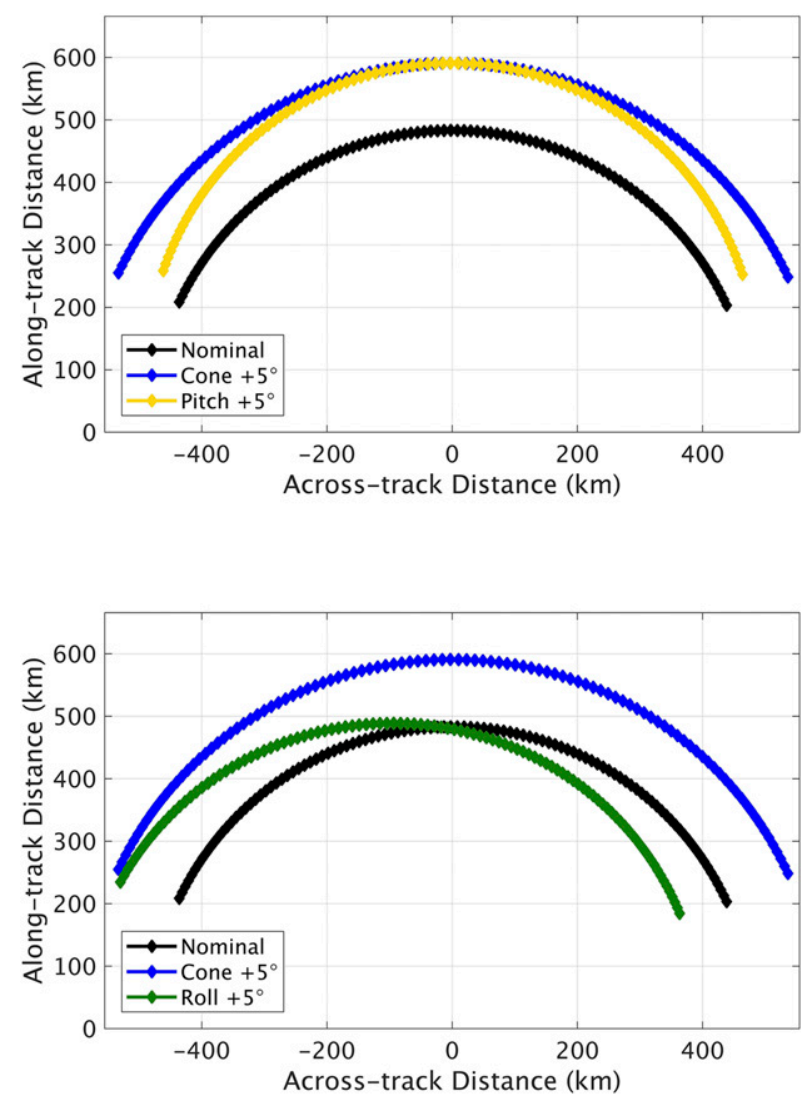

FIG. 6. Modeled effect of a cone angle (blue), pitch (yellow), and roll (green) offset on the TMI scan geolocation from nominal (black), assuming the TRMM spacecraft is located at $(0,0)$ and the center of the TMI swath is looking forward. The degree of offset is exaggerated to see effect. This behavior across the scan is used to distinguish a pitch and roll offset from a cone angle offset.

iterative. Initial values for cone and azimuth angles derived from geolocation difference mapping were used prior to closer inspection of along-scan antenna temperature biases. Patterns seen in these biases led to the new approach of geolocation difference mapping partitioned into three parts of the swath. In this section we use the methods described in the previous section to derive the instrument pitch and roll offsets, recalculate the cone and azimuth angles using the pitch and roll offsets, and compute the new along-scan bias correction based on the updated alignment.

\section{a. Instrument pitch and roll offsets}

Figure 7 shows the cone angle calculated for each subset of the scan for all channels. Two important observations can be made from this figure: 1 ) the $10-\mathrm{GHz}$ channels differ significantly from the $19-85-\mathrm{GHz}$ channels, which is attributed to the two feedhorns; and 2) there is a difference in the cone angle value across the

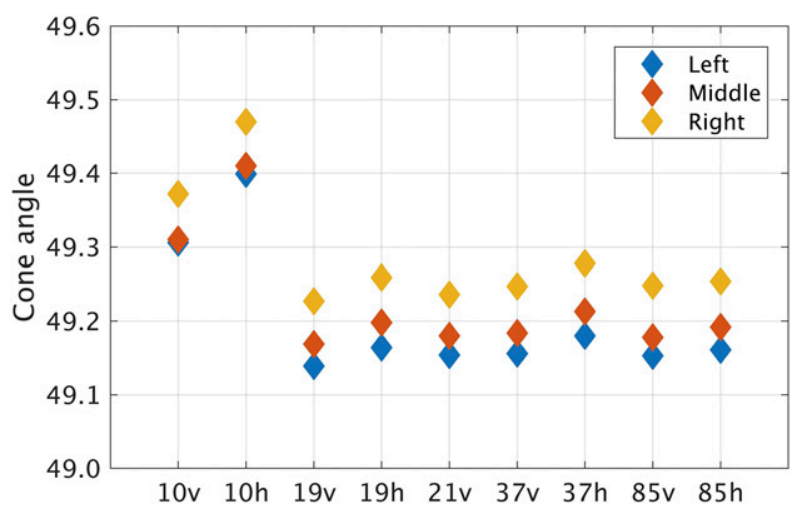

FIG. 7. Cone angle derivation at left (blue), middle (orange) and right (yellow) portions of the TMI scan. The consistent difference between the scans at all channels suggests a pitch and/or roll correction is needed.

scan that is relatively consistent for each feedhorn. For the pitch and roll calculation, we are concerned with observation 2. Note that the left, middle, and right subsets show very similar separations in their feedhorn groups even while the mean values vary per channel. The left-right spread indicates the need for a roll correction, and the off-center middle result indicates the need for a pitch correction.

The derived cone angle across the scan is a function of azimuth, pitch, and roll angles. The pitch and roll offsets that result in the best-fit line to the cone angles of the three scan subsets are taken as the pitch and roll estimates for that channel. This best-fit line is iteratively derived using a Newton-Raphson technique and takes only two iterations to converge. The root-mean-square of the cone angles with the cone angle fits is less than $0.005^{\circ}$ for all channels. Figure 8 shows this best-fit line for the 19v channel, and Fig. 9 gives the pitch and roll derived for all channels using this method. We do not expect the pitch or roll bias from instrument mounting offsets to be different for each channel; therefore, we choose the mean value of $-0.08^{\circ}$ for both pitch and roll. There is some uncertainty associated with this calculation because of the limited data used and the geolocation method. We used the 3-month test dataset described in section 4a for analysis. We also looked at other periods early and late in TRMM for validation and confirmed our results are consistent. The values calculated in Fig. 9 are from the South America region (Fig. 1) and a region that includes Indonesia to utilize more data. If just one of these regions is chosen rather than both, then the pitch and roll offsets derived are slightly different. In addition to this uncertainty, Fig. 9 shows a spread for pitch and roll by channel. The spread in roll by channel is smaller than pitch, giving more 


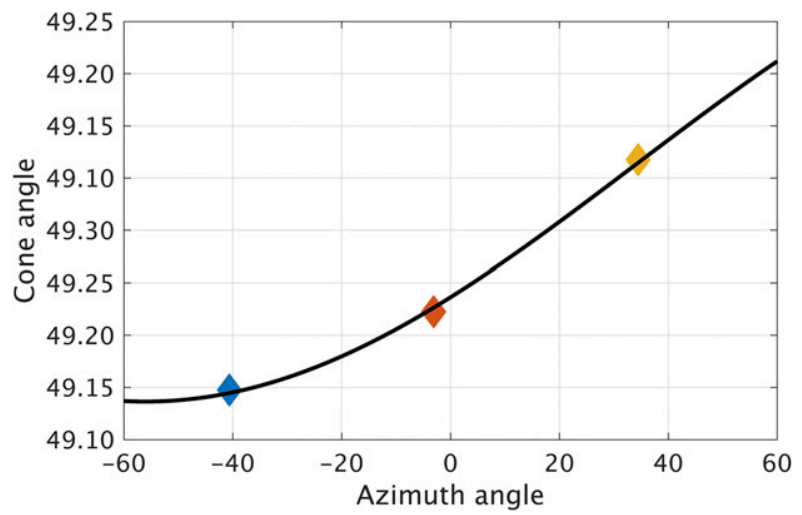

FIG. 8. The variation in the cone angle across the scan is used to derive a pitch-roll angle by fitting a curve of the off-nadir angle as a function of azimuth angle. The curve for $19 \mathrm{v}$ is shown here as an example. Colors correspond to the left (blue), middle (orange), and right (yellow) portions of the TMI scan.

confidence in its accuracy. It is not surprising that the pitch is less certain, given how it is picked out from the small curvature of the scan angle dependence. Based on the standard deviation from different regions and channels, we estimate the uncertainty in the pitch and roll estimates to be approximately $0.03^{\circ}$ and $0.01^{\circ}$, respectively.

\section{b. Cone and azimuth angles}

The new pitch and roll offsets are input into the TMI dataset to recalculate latitude and longitude. The foreaft geolocation difference analysis is then applied using the pitch-roll offset of $-0.08^{\circ}$ to rederive the cone and azimuth angles. The results are shown in Fig. 10 for all channels. As noted previously, there is a significant difference between the $10-\mathrm{GHz}$ channels and the $19-85-\mathrm{GHz}$ channels as a result of the different feedhorns. There is also a noticeable difference between the $10 \mathrm{v}$ and $10 \mathrm{~h}$ cone angles. We believe that this difference is real, as it is probably due to polarization phase center offsets in the feedhorn. Because of feedhorn design constraints, small phase center offsets may exist among channels contained in the same feedhorn (C. S. Ruf 2018, personal communication). One solution to align the beams in a multifrequency feedhorn is described in Imbriale (2006). We do not have information on what tests were performed on TMI to determine alignment of the phase centers, but there was some indication in the ground measurements of a difference in the $10-\mathrm{GHz}$ v- and h-pol cone angles (Shiue 1997). We decided to include this cone angle difference for the calculation of EIA, since EIA accuracy is important in modeling the brightness temperatures. This results in separate EIAs reported for the $10 \mathrm{v}$ and $10 \mathrm{~h}$ channels. However, only one geolocation is reported for both $10-\mathrm{GHz}$ channels

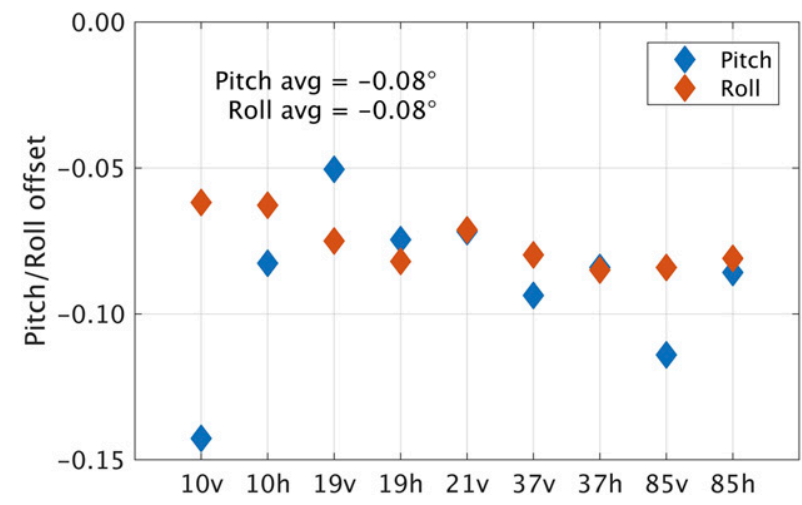

FIG. 9. Derived pitch (blue) and roll (orange) offsets for all channels. The average offset is $-0.08^{\circ}$ for both pitch and roll.

by using the average of the calculated $10 \mathrm{v}$ and $10 \mathrm{~h}$ cone angles. This simplifies the data structure and causes an approximate geolocation error on the order of only $1 \mathrm{~km}$, which was deemed acceptable, since the lowfrequency pixel sizes are over $40 \mathrm{~km}$. The EIAs for the $10 \mathrm{v}$ and $10 \mathrm{~h}$ cone angles are calculated using a cone angle offset from the averaged cone angle. This offset is negative for $10 \mathrm{v}$ and positive for $10 \mathrm{~h}$. For all other channels a single EIA is reported, since the difference in our estimates per channel is not considered significant.

Table 1 shows the average cone and azimuth start angles for the two feedhorns and the cone angle offset used to calculate EIA for the $10-\mathrm{GHz}$ channels. The constants derived by Wentz (2015) are also reported, and we show very similar results. The differences can be attributed to variations in our methods and different spacecraft and instrument attitude calculations. As with the pitch-roll geolocation analysis, we used the 3-month test dataset to derive the cone and azimuth angles and then confirmed that the cone and azimuth angles remained constant throughout TRMM's lifetime. Figure 11 shows the updated TMI yaw difference maps using the new instrument pitch and roll offsets and the cone and azimuth angles from Table 1 for 2004. The alignment of all channels is greatly improved using the new constants compared to Fig. 1. Residual effects over the Andes still exist, which may be caused by fore/aft sampling variations of the surface snow cover and the lack of topography in the geolocation algorithm. For example, Lake Titicaca at an altitude of $3.8 \mathrm{~km}$ shows clear coastline errors.

\section{c. Along-scan bias correction}

Once the pitch-roll offset is derived and the cone angle and azimuth angles are updated, the along-scan bias correction analysis can be reapplied. Figure 12 shows the new along-scan bias at both cold and warm 

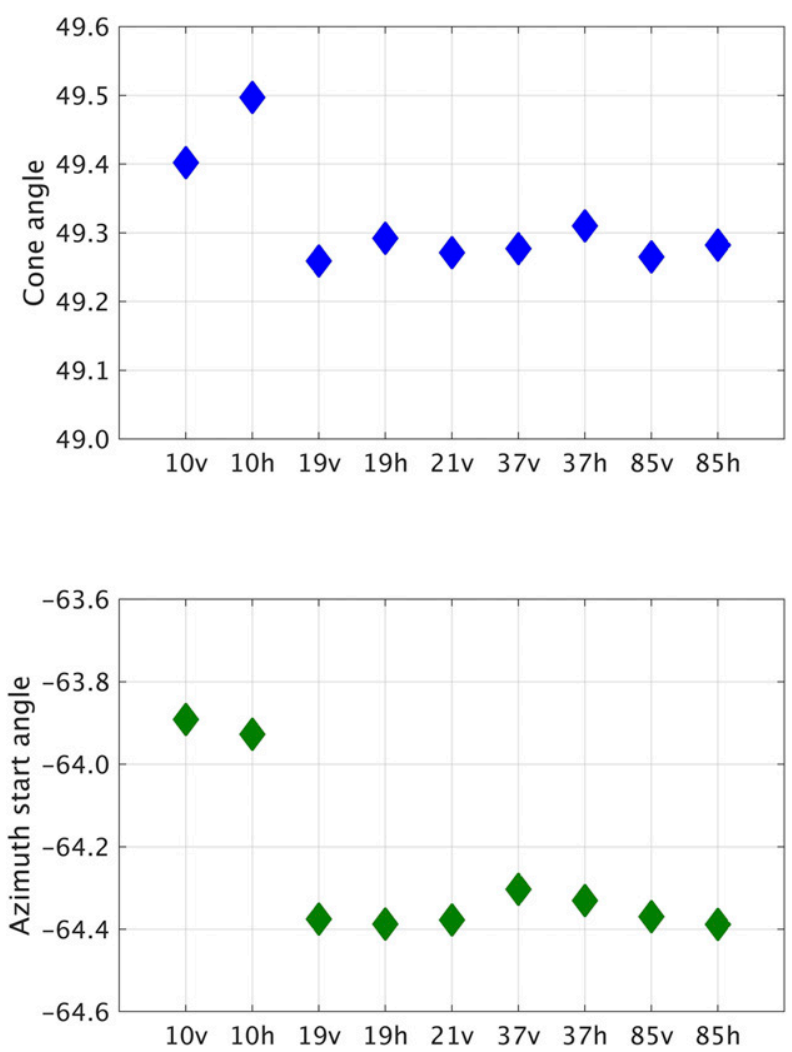

FIG. 10. TMI (top) cone and (bottom) azimuth angles derived from geolocation analysis. The two feedhorns give significantly different results.

TAs compared with V7. The V7 biases are the same for both cold and warm TAs, since there is a constant additive bias regardless of scene temperature. The V8 along-scan biases are derived using the vicarious cold and warm techniques, accounting for the pitch-roll offset and calculating a bias at a given scene temperature according to Eq. (1). As seen in Fig. 12, the V8 cold and warm biases have greater agreement for the middle part of the scan compared to Fig. 4, giving us confidence that the correct pitch/roll offset has been applied. Figure 12 also shows the impact of using both cold and warm biases for correcting edge-of-scan obstructions. Version 7 assumed these biases were constant as a function of scene temperature, so it did not capture the temperaturedependent scan biases near the edge of the scan. Version 8 differs from V7 at the edge of the scan for most channels but particularly for $19 \mathrm{~h}$ and $37 \mathrm{~h}$, which differ by about $1 \mathrm{~K}$ at the right side of the scan.

\section{Summary}

The TRMM data products from PPS have been updated to a final version 8 following the decommissioning of the TRMM spacecraft in April 2015 after a very successful 17-plus years of operation. This final version includes several corrections to TMI to improve the calibration and dataset quality. This paper described two improvements to the TMI dataset for V8: sensor alignment and along-scan bias corrections.

The sensor alignment was analyzed by creating geolocation maps of the antenna temperature difference between TMI forward and backward looks at coastlines. Prior versions used at-launch values for alignment that were found to not be correct. Using the geolocation maps, we updated the alignment to include an instrument pitch and roll offset of $-0.08^{\circ}$ and $-0.08^{\circ}$, respectively, and we modified the cone and azimuth start angles to account for differences in the two feedhorns.

The along-scan bias correction was updated to include the effects of temperature-dependent edge-of-scan obstruction and the new instrument pitch and roll offset. Previous TMI versions used over-ocean TA biases to correct for along-scan biases, but we found this to be inadequate at all scene temperatures. Therefore, we used a linear interpolation between cold and warm TA references to derive a new correction for V8. The updated along-scan bias correction is very similar to the V7 correction with the large- and small-scale fluctuations, but there are two main differences: the slight slope adjustment due to the inclusion of a pitch and roll offset, and the edge-of-scan differences resulting from the inclusion of the warm TA reference.

The two updates described here, along with the other updates for TMI V8, significantly improve the PPSreleased TMI dataset. The alignment improvements do not directly affect the brightness temperatures; however, they do impact geolocation and the earth incidence angles, which are necessary to accurately model the TBs in retrieval algorithms. The along-scan bias correction directly impacts the TBs, and the effect on retrieval algorithms will be most pronounced near the edge of the

TABLE 1. Cone angles and azimuth start angles calculated for TMI V8 data release, comparing the values derived here (V8) with Wentz (2015).

Channel Cone angle (V8) Cone angle offset (V8) Cone angle (Wentz) Azimuth start angle (V8) Azimuth start angle (Wentz)

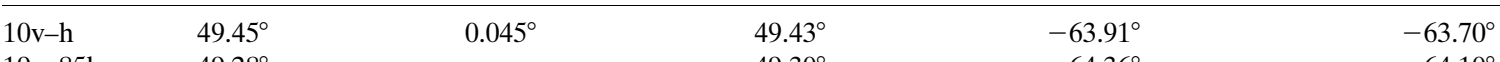

$\begin{array}{llllll}19 \mathrm{v}-85 \mathrm{~h} & 49.28^{\circ} & - & 49.30^{\circ} & -64.36^{\circ} & -64.10^{\circ}\end{array}$



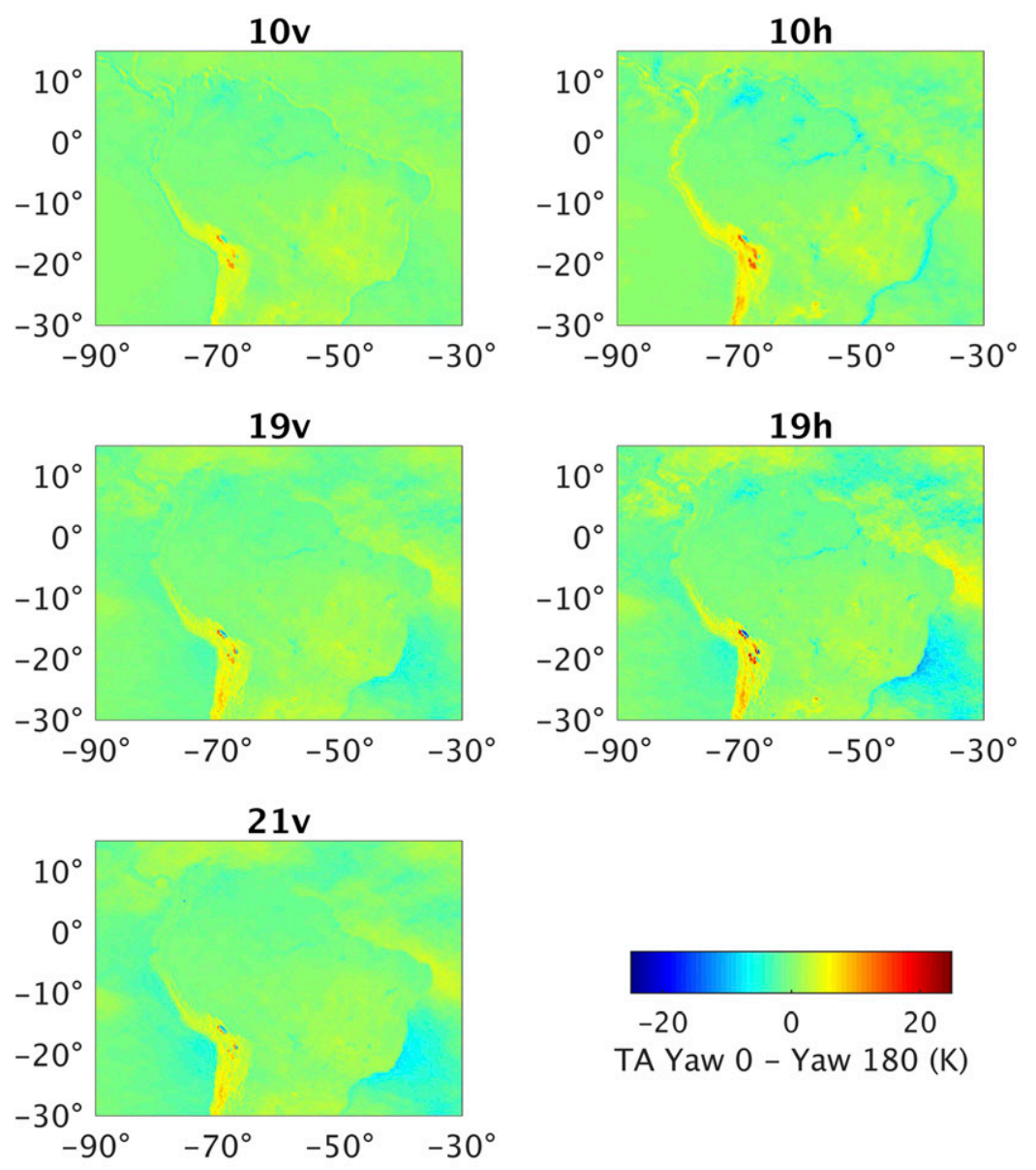

$37 v$
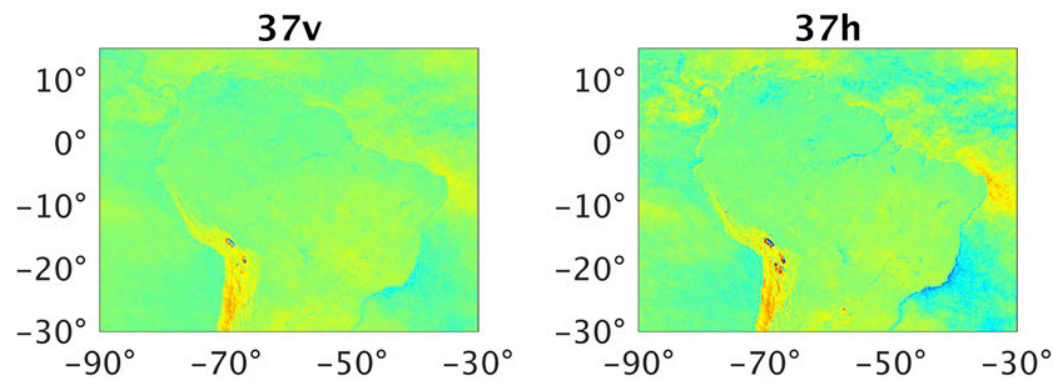

$85 v$
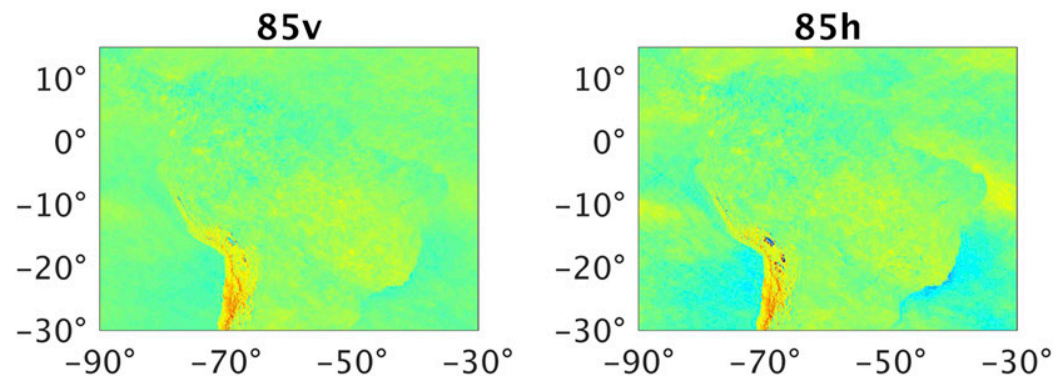

FIG. 11. Yaw 0 - Yaw 180 gridded TMI TAs over South America for updated instrument attitude and cone and azimuth angles to be used in V8: (top left) 10v to (bottom left) 21v. The geolocation for all channels is greatly improved compared to Fig. 1. 
10v

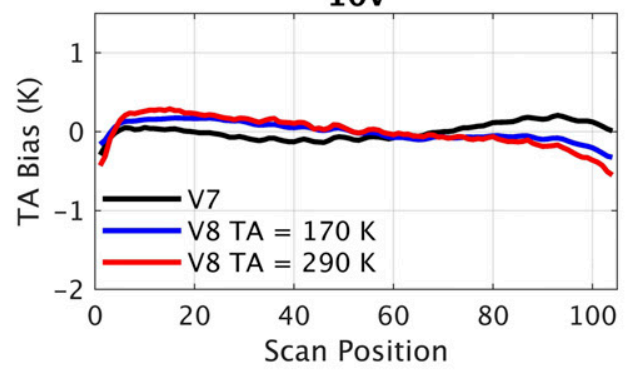

$19 v$

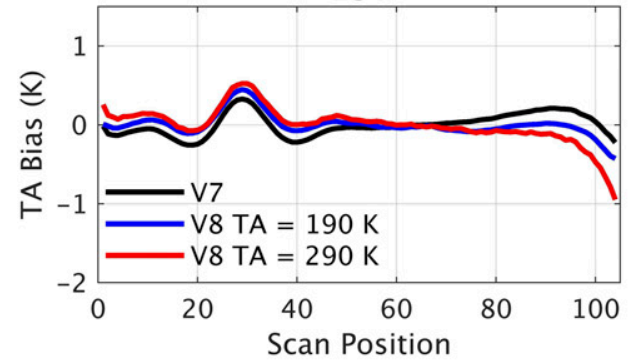

21v

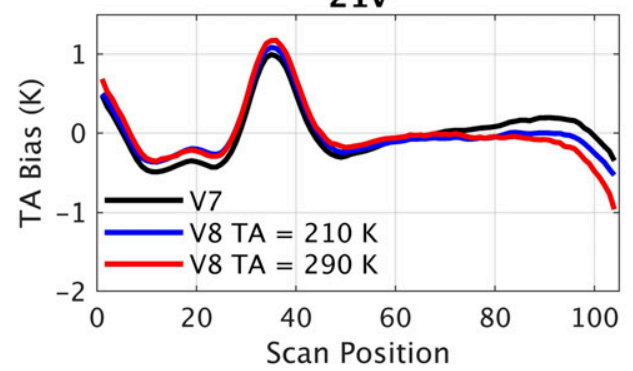

$37 v$

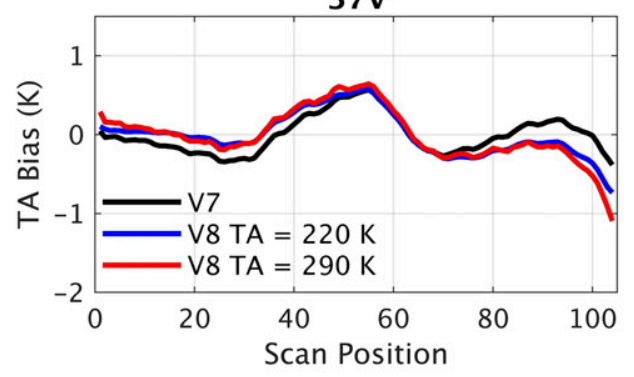

85v

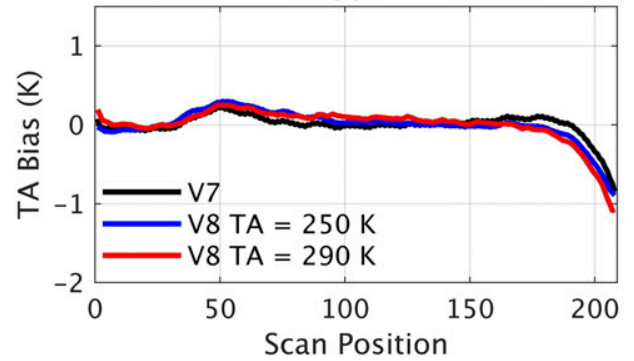

10h

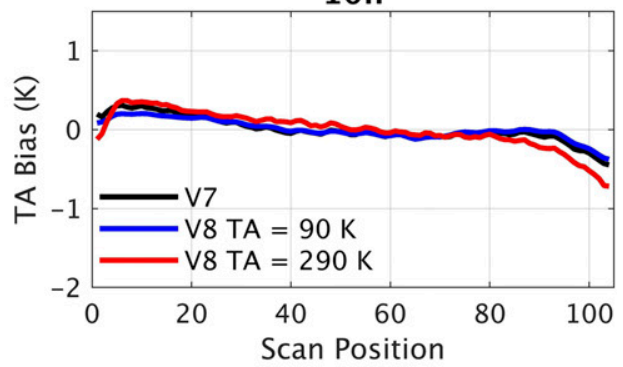

19h

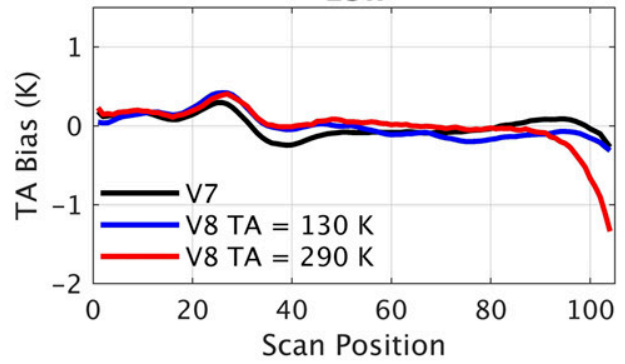

$37 \mathrm{~h}$

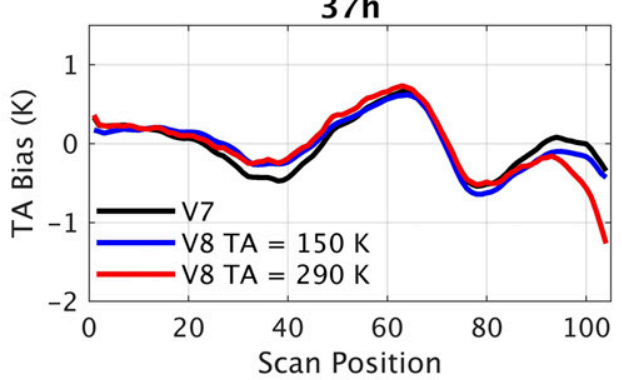

85h

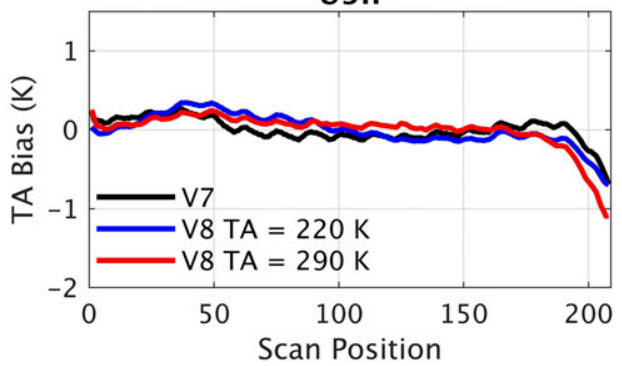

FIG. 12. Comparison of V7 (black), V8 cold (blue), and V8 warm (red) estimates of TMI scan biases for (top left) 10v to (bottom left) 21v. The V7 method assumes these biases are constant as a function of scene temperature, so the new biases can differ significantly at the edge of scan (e.g., $\sim 1 \mathrm{~K}$ right side of $19 \mathrm{~h}$ scan). The pitch-roll offset included in V8 results in greater agreement between cold and warm biases for the middle of the scan. 
scan, where the updated correction adjusts the TBs at warm scene temperatures by nearly $1 \mathrm{~K}$ for some channels. The alignment and along-scan bias improvements ensure an accurate TMI observational dataset that can be reliably used in retrieval algorithms and for studying long-term geophysical trends.

Acknowledgments. Funding for this work was provided by NASA's GPM mission and the NASA Precipitation Measurement Missions (PMM) science team under Grant NNX16AE38G.

\section{REFERENCES}

Alquaied, F., R. Chen, and W. L. Jones, 2018a: Hot load temperature correction for TRMM Microwave Imager in the legacy brightness temperature. IEEE J. Sel. Topics Appl. Earth Obs. Remote Sens., https://doi.org/10.1109/JSTARS.2018.2837099, in press.

,$- \ldots$, and $-2018 \mathrm{~b}$ : Emissive reflector correction in the legacy version 1B11 V8 (GPM05) brightness temperature of the TRMM Microwave Imager. IEEE J. Sel. Top. Appl. Earth Obs. Remote Sens., https://doi.org/10.1109/JSTARS.2018.2797021, in press.

Berg, W., 2017: Towards developing a long-term high-quality intercalibrated TRMM/GPM radiometer dataset. 2017 IEEE International Geoscience and Remote Sensing Symposium: Proceedings, IEEE, 248-250, https://doi.org/10.1109/ IGARSS.2017.8126941.

- M. R. P. Sapiano, J. Horsman, and C. Kummerow, 2013: Improved geolocation and earth incidence angle information for a fundamental climate data record of the SSM/I sensors. IEEE Trans. Geosci. Remote Sens., 51, 1504-1513, https:// doi.org/10.1109/TGRS.2012.2199761.

— - and Coauthors, 2016: Intercalibration of the GPM microwave radiometer constellation. J. Atmos. Oceanic Technol., 33, 2639-2654, https://doi.org/10.1175/JTECH-D-16-0100.1.

Draper, D. W., 2016: Calibration data book for Global Precipitation Measurement (GPM) Microwave Imager (GMI). Revision H, Ball Aerospace and Technologies Corp. Doc. 2344649, 229 pp. , 2018: Radio frequency environment for Earth-observing passive microwave imagers. IEEE J. Sel. Top. Appl. Earth Obs. Remote Sens., https://doi.org/10.1109/JSTARS.2018.2801019, in press.

- D. A. Newell, F. J. Wentz, S. Krimchansky, and G. M. Skofronick-Jackson, 2015: The Global Precipitation Measurement (GPM) Microwave Imager (GMI): Instrument overview and early on-orbit performance. IEEE J. Sel. Top. Appl. Earth Obs. Remote Sens., 8, 3452-3462, https://doi.org/ 10.1109/JSTARS.2015.2403303.

Gopalan, K., W. L. Jones, S. Biswas, S. Bilanow, T. Wilheit, and T. Kasparis, 2009: A time-varying radiometric bias correction for the TRMM Microwave Imager. IEEE Trans. Geosci. Remote Sens., 47, 3722-3730, https://doi.org/ 10.1109/TGRS.2009.2028882.

Hollinger, J. P., J. L. Peirce, and G. A. Poe, 1990: SSM/I instrument evaluation. IEEE Trans. Geosci. Remote Sens., 28, 781-790, https://doi.org/10.1109/36.58964.
Hou, A. Y., and Coauthors, 2014: The Global Precipitation Measurement Mission. Bull. Amer. Meteor. Soc., 95, 701-722, https://doi.org/10.1175/BAMS-D-13-00164.1.

Imbriale, W. A., Ed., 2006: Spaceborne Antennas for Planetary Exploration. JPL Deep Space Communications and Navigation Series, Vol. 12, John Wiley \& Sons, 592 pp.

Kroodsma, R. A., D. S. McKague, and C. S. Ruf, 2012: Satellite attitude analysis using the vicarious cold calibration method for microwave radiometers. 2012 IEEE International Geoscience and Remote Sensing Symposium: Proceedings, IEEE, 2964-2967, https://doi.org/10.1109/IGARSS.2012.6350804.

$\longrightarrow, \ldots$, and $\longrightarrow, 2017$ : Vicarious cold calibration for conical scanning microwave imagers. IEEE Trans. Geosci. Remote Sens., 55, 816-827, https://doi.org/10.1109/TGRS.2016.2615552.

Kummerow, C., W. Barnes, T. Kozu, J. Shiue, and J. Simpson, 1998: The Tropical Rainfall Measuring Mission (TRMM) sensor package. J. Atmos. Oceanic Technol., 15, 809-817, https:// doi.org/10.1175/1520-0426(1998)015<0809:TTRMMT>2.0.CO;2.

Kunkee, D. B., G. A. Poe, D. J. Boucher, S. D. Swadley, Y. Hong, J. E. Wessel, and E. A. Uliana, 2008: Design and evaluation of the first special sensor microwave imager/sounder. IEEE Trans. Geosci. Remote Sens., 46, 863-883, https://doi.org/ 10.1109/TGRS.2008.917980.

McKague, D. S., C. S. Ruf, and J. J. Puckett, 2011: Beam spoiling correction for spaceborne microwave radiometers using the twopoint vicarious calibration method. IEEE Trans. Geosci. Remote Sens., 49, 21-27, https://doi.org/10.1109/TGRS.2010.2068052.

Moradi, I., H. Meng, R. R. Ferraro, and S. Bilanow, 2013: Correcting geolocation errors for microwave instruments aboard NOAA satellites. IEEE Trans. Geosci. Remote Sens., 51, 3625-3637, https://doi.org/10.1109/TGRS.2012.2225840.

Poe, G. A., E. A. Uliana, B. A. Gardiner, T. E. vonRentzell, and D. B. Kunkee, 2008: Geolocation error analysis of the Special Sensor Microwave Imager/Sounder. IEEE Trans. Geosci. Remote Sens., 46, 913-922, https://doi.org/10.1109/ TGRS.2008.917981.

Shiue, J., 1997: TMI alignment and other coefficients for the TMI database at TRMM. NASA Internal Memo., 6 pp.

Stocker, E. F., F. Alquaied, S. Bilanow, Y. Ji, and L. Jones, 2018: TRMM version 8 reprocessing improvements and incorporation into the GPM data suite. J. Atmos. Oceanic Technol., 35, 1181-1199, https://doi.org/10.1175/JTECH-D-17-0166.1.

Wentz, F. J., 2015: A 17-yr climate record of environmental parameters derived from the Tropical Rainfall Measuring Mission (TRMM) Microwave Imager. J. Climate, 28, 6882-6902, https://doi.org/10.1175/JCLI-D-15-0155.1.

- , and D. Draper, 2016: On-orbit absolute calibration of the Global Precipitation Measurement Microwave Imager. J. Atmos. Oceanic Technol., 33, 1393-1412, https://doi.org/ 10.1175/JTECH-D-15-0212.1.

— , P. Ashcroft, and C. Gentemann, 2001: Post-launch calibration of the TRMM Microwave Imager. IEEE Trans. Geosci. Remote Sens., 39, 415-422, https://doi.org/10.1109/36.905249.

Yang, J. X., D. S. McKague, and C. S. Ruf, 2016: Boreal, temperate, and tropical forests as vicarious calibration sites for spaceborne microwave radiometry. IEEE Trans. Geosci. Remote Sens., 54, 1035-1051, https://doi.org/10.1109/ TGRS.2015.2472532. 